\title{
Erratum to: Mitochondrial CHCHD-Containing Proteins: Physiologic Functions and Link with Neurodegenerative Diseases
}

Zhi-Dong Zhou ${ }^{1,2} \cdot$ Wuan-Ting Saw ${ }^{1} \cdot$ Eng-King Tan ${ }^{1,2,3}$

Published online: 7 October 2016

(C) Springer Science+Business Media New York 2016

\section{Erratum to: Mol Neurobiol}

DOI 10.1007/s12035-016-0099-5

The original version of this article unfortunately contained mistakes and the authors hereby publishing these corrections. The reference citations on Table 2 were incorrect in both pdf and HTML versions of the original article. The corrected Table 2 is given below.

Another error in reference citation appears in the subsection "Transcriptional Modulation of Gene Expressions by CHCHD Proteins". In the sentence "Furthermore, CHCHD3 is associated with the regulation of expression of an anti-apoptotic protein, BAG-1 [31].", the citation was incorrectly referred to Ajroud-Driss et al. (2015). The proper reference citation should be with Liu et al. (2012) [15] to read "Furthermore, CHCHD3 is associated with the regulation of expression of an anti-apoptotic protein, BAG-1 [15]."

With these, the original article was corrected.

The corresponding references are also given herewith.

The online version of the original article can be found at http://dx.doi. org/10.1007/s12035-016-0099-5

Zhi-Dong Zhou

Zhidong_zhou@nni.com.sg

Eng-King Tan

Tan.eng.king@sgh.com.sg

Wuan-Ting Saw

saw.wuan.ting@singhealth.com.sg

1 National Neuroscience Institute of Singapore, 11 Jalan Tan Tock Seng, Singapore 308433, Singapore

2 Signature Research Program in Neuroscience and Behavioural Disorders, Duke-NUS Graduate Medical School Singapore, 8 College Road, Singapore 169857, Singapore

3 Department of Neurology, Singapore General Hospital, Outram Road, Singapore 169608, Singapore 
Table 2 Gene variants of CHCHD10 identified in human neurological disorders

\begin{tabular}{|c|c|c|c|c|c|}
\hline Gene variants & Amino acid changes & Relevant human diseases & Onset age & Case no. & Refs \\
\hline c. $34 \mathrm{C}>\mathrm{T}$ & p. Pro12Ser & ALS & 58 & 1 & {$[94]$} \\
\hline c. $43 \mathrm{C}>\mathrm{A}$ & p. Arg15Ser & $\begin{array}{l}\text { Autosomal dominant } \\
\text { mitochondrial myopathy }\end{array}$ & $?$ & 1 & {$[31]$} \\
\hline \multirow[t]{2}{*}{ c. $44 \mathrm{C}>\mathrm{A}$} & \multirow[t]{2}{*}{ p. Arg15Leu } & Familial ALS & $35-73$ & 13 & {$[93,96]$} \\
\hline & & Sporadic ALS & 54 & 1 & {$[95]$} \\
\hline c. $64 \mathrm{C}>\mathrm{T}$ & p. His22Tyr & Sporadic FTD & 54 & 1 & [97] \\
\hline c. $67 \mathrm{C}>\mathrm{A}$ & p. Pro23Thr & Familial FTD & 50 & 1 & {$[95]$} \\
\hline c. $67 \mathrm{C}>\mathrm{T}$ & p. Pro23Ser & Sporadic FTD & 66 & 1 & [97] \\
\hline c. $68 \mathrm{C}>\mathrm{T}$ & p. Pro23Leu & Sporadic FTD & 52 & 1 & [97] \\
\hline c. $95 \mathrm{C}>\mathrm{A}$ & p. Ala32Asp & Sporadic FTD & 76 & 1 & [97] \\
\hline \multirow{4}{*}{$\begin{array}{l}\text { c. } 100 \mathrm{C}>\mathrm{T} \\
\quad(\mathrm{rs} 551521196)\end{array}$} & \multirow[t]{4}{*}{ p. Pro34Ser } & ALS/FTD & $59-67$ & 2 & [92] \\
\hline & & Sporadic ALS & $44-75$ & 4 & {$[98,99]$} \\
\hline & & $\mathrm{PD}$ & $?$ & 1 & {$[95]$} \\
\hline & & Alzheimer's disease & $?$ & 2 & [95] \\
\hline c. $104 \mathrm{C}>\mathrm{A}$ & p. Ala35Asp & Sporadic FTD & 51 & 1 & [95] \\
\hline c. $170 \mathrm{~T}>\mathrm{A}$ & p. Val57Glu & Sporadic FTD & 60 & 1 & [97] \\
\hline c. $172 \mathrm{G}>\mathrm{C}$ & p. Gly58Arg & $\begin{array}{l}\text { Autosomal dominant } \\
\text { mitochondrial myopathy }\end{array}$ & $?$ & 1 & {$[31]$} \\
\hline c. $176 \mathrm{C}>\mathrm{T}$ & p. Ser59Leu & $\begin{array}{l}\text { ALS/FTD, cerebellar sign, } \\
\text { Parkinsonism }\end{array}$ & $49-67$ & 9 & {$[8,92]$} \\
\hline \multirow[t]{3}{*}{ c. $197 \mathrm{G}>\mathrm{T}$} & \multirow[t]{3}{*}{ p. Gly66Val } & Familial ALS & $35-73$ & 1 & {$[96]$} \\
\hline & & $\begin{array}{l}\text { Spinal muscular atrophy } \\
\text { (SMAJ) }\end{array}$ & $14-72$ & 55 & [100] \\
\hline & & $\begin{array}{l}\text { Charcot-Marie-Tooth } \\
\text { neuropathy (CMT2) }\end{array}$ & $30-55$ & 12 & [101] \\
\hline \multirow[t]{2}{*}{ c. $239 \mathrm{C}>\mathrm{T}$} & \multirow[t]{2}{*}{ p. Pro80Leu } & Sporadic ALS & $25-59$ & 3 & {$[95,99]$} \\
\hline & & Familial ALS & 43 & 1 & {$[95]$} \\
\hline c. $244 \mathrm{C}>\mathrm{T}$ & p. Glu82X & FTD & 58 & 1 & [94] \\
\hline
\end{tabular}

\section{References}

15. Liu H, Li Y, Li Y, Liu B, Wu H, Wang J, Wang Y, Wang M et al (2012) Cloning and functional analysis of FLJ20420: a novel transcription factor for the BAG-1 promoter. PLoS One 7(5):e34832. doi:10.1371/journal.pone. 0034832

31. Ajroud-Driss S, Fecto F, Ajroud K, Lalani I, Calvo SE, Mootha VK, Deng HX, Siddique N et al (2015) Mutation in the novel nuclear-encoded mitochondrial protein CHCHD10 in a family with autosomal dominant mitochondrial myopathy. Neurogenetics 16(1):1-9. doi:10.1007/s10048-014-0421-1

92. Chaussenot A, Le Ber I, Ait-El-Mkadem S, Camuzat A, de Septenville A, Bannwarth S, Genin EC, Serre V et al (2014) Screening of CHCHD10 in a French cohort confirms the involvement of this gene in frontotemporal dementia with amyotrophic lateral sclerosis patients. Neurobiology of aging 35(12):2884e2881-2884. doi:10.1016/j.neurobiolaging.2014.07.022
93. Johnson JO, Glynn SM, Gibbs JR, Nalls MA, Sabatelli M, Restagno G, Drory VE, Chio A et al (2014) Mutations in the CHCHD10 gene are a common cause of familial amyotrophic lateral sclerosis. Brain: A Journal of Neurology 137(Pt 12):e311. doi:10.1093/brain/awu265

94. Dols-Icardo O, Nebot I, Gorostidi A, Ortega-Cubero S, Hernandez I, Rojas-Garcia R, Garcia-Redondo A, Povedano $M$ et al (2015) Analysis of the CHCHD10 gene in patients with frontotemporal dementia and amyotrophic lateral sclerosis from Spain. Brain: A Journal of Neurology 138(Pt 12): e400. doi:10.1093/brain/awv175

95. Zhang M, Xi Z, Zinman L, Bruni AC, Maletta RG, Curcio SA, Rainero I, Rubino E et al (2015) Mutation analysis of CHCHD10 in different neurodegenerative diseases. Brain: A Journal of Neurology 138(Pt 9):e380. doi:10.1093/brain/awv082

96. Muller K, Andersen PM, Hubers A, Marroquin N, Volk AE, Danzer KM, Meitinger T, Ludolph AC et al (2014) Two novel mutations in conserved codons indicate that $\mathrm{CHCHD} 10$ is a gene 
associated with motor neuron disease. Brain: A Journal of Neurology 137(Pt 12):e309. doi:10.1093/brain/awu227

97. Jiao B, Xiao T, Hou L, Gu X, Zhou Y, Zhou L, Tang B, Xu J et al (2016) High prevalence of CHCHD10 mutation in patients with frontotemporal dementia from China. Brain: A Journal of Neurology 139(Pt 4):e21. doi:10.1093 /brain/awv367

98. Chio A, Mora G, Sabatelli M, Caponnetto C, Traynor BJ, Johnson JO, Nalls MA, Calvo A et al (2015) CHCH10 mutations in an Italian cohort of familial and sporadic amyotrophic lateral sclerosis patients. Neurobiology of Aging 36(4):1767.e1763-1766. doi:10.1016/j.neurobiolaging.2015.01.017
99. Ronchi D, Riboldi G, Del Bo R, Ticozzi N, Scarlato M, Galimberti D, Corti S, Silani V et al (2015) CHCHD10 mutations in Italian patients with sporadic amyotrophic lateral sclerosis. Brain: A Journal of Neurology 138(Pt 8):e372. doi:10.1093/brain/awu384

100. Penttila S, Jokela M, Bouquin H, Saukkonen AM, Toivanen J, Udd B (2015) Late onset spinal motor neuronopathy is caused by mutation in CHCHD10. Annals of neurology 77(1):163-172. doi:10.1002/ana.24319

101. Auranen M, Ylikallio E, Shcherbii M, Paetau A, Kiuru-Enari S, Toppila JP, Tyynismaa H (2015) CHCHD10 variant p.(Gly66Val) causes axonal Charcot-Marie-Tooth disease. Neurol Genet 1(1): e1. doi:10.1212/NXG.0000000000000003 